\title{
On the correlation between the binding energies of the triton and the $\alpha$-particle
}

\author{
L. Plattel* \\ Forschungszentrum Jülich, Institut für Kernphysik, D-52425 Jülich, Germany and \\ Helmholtz-Institut für Strahlen- und Kernphysik (Theorie), \\ Universität Bonn, Nußallee 14-16, D-53115 Bonn, Germany \\ H.-W. Hammer \\ Institute for Nuclear Theory, University of Washington, Seattle, WA 98195, USA \\ Ulf-G. Meißner用 \\ Helmholtz-Institut für Strahlen- und Kernphysik (Theorie), \\ Universität Bonn, Nußallee 14-16, D-53115 Bonn, Germany and \\ Forschungszentrum Jülich, Institut für Kernphysik, D-52425 Jülich, Germany
}

(Dated: November 5, 2018)

\begin{abstract}
We consider the correlation between the binding energies of the triton and the $\alpha$-particle which is empirically observed in calculations employing different phenomenological nucleon-nucleon interactions. Using an effective quantum mechanics approach for short-range interactions with large scattering length $|a| \gg \ell$, where $\ell$ is the natural low-energy length scale, we construct the effective interaction potential at leading order in $\ell /|a|$. In order to renormalize the four-nucleon system, it is sufficient to include a $S U(4)$-symmetric one-parameter three-nucleon interaction in addition to the S-wave nucleon-nucleon interactions. The absence of a four-nucleon force at this order explains the empirically observed correlation between the binding energies of the triton and the $\alpha$-particle. We calculate this correlation and obtain a prediction for the $\alpha$-particle binding energy. Corrections to our results are suppressed by $\ell /|a|$.
\end{abstract}

PACS numbers: 21.45.+v, 21.10.Dr, 03.65.Ge

*Electronic address: 1.platter@fz-juelich.de

${ }^{\dagger}$ Electronic address: hammer@phys.washington.edu

${ }^{\ddagger}$ Electronic address: meissner@itkp.uni-bonn.de 
The scattering of two particles with short-range interactions at sufficiently low energy is determined by their S-wave scattering length $a$. Generically, the scattering length $a$ is comparable to the typical low-energy length scale of the system $\ell$ which, for short-range interactions, is of the order of the range of the potential. In special cases, however, the scattering length can be much larger, $|a| \gg \ell$, due to a fine-tuning of the parameters in the underlying interaction potential. Such a fine-tuning can be accidental as in the case of two nucleons at low energy, or it can be controlled experimentally by varying an external parameter such as a magnetic field in the case of Feshbach resonances for atomic systems.

Few-body systems with large scattering length are particularly interesting because such systems show many universal properties. The simplest example for positive $a$ is the existence of a shallow bound state (dimer) with binding energy

$$
B_{d}=\frac{\hbar^{2}}{m a^{2}}+\mathcal{O}(\ell / a), \quad a>0,
$$

where $m$ is the mass of the particles. The separation of scales between $|a|$ and $\ell$ also manifests itself in few-body systems with more than two particles. The most prominent example is the Efimov effect: the occurence of a geometric spectrum of three-body bound states in the limit $a \rightarrow \pm \infty$ [1]. In this limit, the ratio of the binding energies of successive Efimov states approaches the universal number 515.03... In order to fully specify the bound state spectrum, however, the absolute energy of one Efimov state has to be specified and this introduces one three-body parameter, $L_{3}$, which will be discussed in more detail below. As a consequence, a three-body system with a large but finite scattering length can be characterized by specifying the values of $a$ and $L_{3}$.

There has been a considerable interest in physical systems with large scattering length recently. This interest was stimulated by the experimental realization of Feshbach resonances with trapped atoms and by the effective field theory program for nuclear physics. (See, e.g., Refs. 2, 3] and references therein.) The best known example of a nuclear system with a large scattering length is the two-nucleon $(N N)$ system. There are two independent S-wave scattering lengths that govern the low-energy scattering of nucleons. The scattering lengths $a_{s}=-23.5 \mathrm{fm}$ and $a_{t}=5.42 \mathrm{fm}$ describe $N N$ scattering in the spin-singlet $\left({ }^{1} S_{0}\right)$ and spintriplet $\left({ }^{3} S_{1}\right)$ channels, respectively. Both scattering lengths are significantly larger than the natural low-energy length scale $\ell \sim \hbar /\left(m_{\pi} c\right) \approx 1.4 \mathrm{fm}$, while the effective ranges are of the same order as $\ell$. As a consequence, an expansion in $\ell /|a|$ is useful.

A peculiar universal feature of the three-nucleon system is the Phillips line [4]. If the predictions of different nucleon-nucleon potentials for the triton binding energy $B_{t}$ and the spin-doublet neutron-deuteron scattering length $a_{n d}^{(1 / 2)}$ are plotted against each other, they fall close to a line. This correlation between $B_{t}$ and $a_{n d}^{(1 / 2)}$ is called the Phillips line and can not be understood in conventional potential models. However, it immediately follows from universality if the large $N N$ scattering lengths are exploited within an expansion in $\ell /|a|$ [5, 6]. If corrections of order $\ell /|a|$ are neglected, all low-energy 3-nucleon observables depend only on the spin-singlet and spin-triplet scattering lengths $a_{s}$ and $a_{t}$ and the three-body parameter $L_{3}$. Since the $N N$ potentials reproduce the scattering phase shifts, they all have the same scattering lengths. The short distance part of the potentials which is encoded in the three-body parameter $L_{3}$, however, is not constrained by the phase shifts and in general is different for each potential. The different potential model calculations must therefore fall close to a line which is parametrized by the parameter $L_{3}$. A similar universal feature of the four-nucleon system is the Tjon line: an approximately linear correlation between 
the triton binding energy $B_{t}$ and the binding energy of the $\alpha$-particle $B_{\alpha}$. This correlation was discovered by Tjon [7] using simple separable interactions, but also holds for modern phenomenological potentials 8]. The origin of this correlation has not been explained. However, it would immediately follow if the four-nucleon system could be renormalized to leading order in $l /|a|$ without specifying a four-body parameter. The purpose of this paper is to study the four-nucleon system in an effective quantum mechanics approach (see, e.g., Ref. 9] ) to leading order in $\ell /|a|$ in order to understand the origin of the Tjon line. We will show that no new four-body parameter is required to renormalize the four-nucleon system and calculate the binding energy of the $\alpha$-particle to leading order in $\ell /|a|$.

Effective theories are a convenient tool to calculate observables of low-energy systems to high precision in a systematic fashion. They are ideally suited to exploit a separation of scales such as the one between $|a|$ and $\ell$. At sufficiently low energy, every interaction can be considered pointlike. One can then use a very general effective theory to describe the universal low-energy properties of the system in which observables are computed with a controlled expansion in $\ell /|a|$. Few-body systems with large scattering length are of special interest: It is known that in the three-body sector with short-range interactions a threebody force at leading order is needed to renormalize the theory [10]. This implies that at leading order in $l /|a|$, the properties of the three-body system with large scattering length are not determined by two-body data alone and one piece of three-body information (such as the three-body binding energy) is required as well. Recently we considered the four-boson system with short-range interactions. We showed that at leading order no four-body force is needed for consistent renormalization and applied the theory to atomic ${ }^{4}$ He clusters [11].

In this letter, we extend this work to the four-nucleon system. There has been a large interest recently in applying effective field theory methods to the few-nucleon problem. (See, e.g., Refs. 3, 12, 13] and references therein.) The four-nucleon problem is more complicated than the four-boson problem due to spin and isospin. Both the ${ }^{1} S_{0}$ and ${ }^{3} S_{1} N N$ scattering lengths $a_{s}$ and $a_{t}$ contribute at leading order in $\ell /|a|$. However, as in the case of bosons, only one three-body parameter $L_{3}$ enters for the $\alpha$ particle. The parameter $L_{3}$ determines the $S U(4)$-symmetric three-nucleon force that is required to renormalize the three-nucleon problem [6]. In the following, we will show that no four-body parameter is required to renormalize the nuclear four-nucleon system and that the binding energy of the $\alpha$-particle can be described to $10 \%$ accuracy at leading order in $\ell /|a|$. Furthermore, we will calculate the Tjon line at leading order in $\ell /|a|$.

The effective low-energy interaction potential generated by short-range contact interactions can be written down in a momentum expansion. In the S-wave sector of the two-nucleon system, it takes the general form

$$
\left\langle\mathbf{k}^{\prime}|V| \mathbf{k}\right\rangle=\mathcal{P}_{s} \lambda_{2}^{s} g\left(\mathbf{k}^{\prime}\right) g(\mathbf{k})+\mathcal{P}_{t} \lambda_{2}^{t} g\left(\mathbf{k}^{\prime}\right) g(\mathbf{k})+\ldots
$$

where the dots indicate momentum dependent terms that are higher order in $\ell /|a|$ and $\mathcal{P}_{s}$ and $\mathcal{P}_{t}$ project onto the ${ }^{1} S_{0}$ and ${ }^{3} S_{1}$ partial waves, respectively. Because of Galilean invariance, the interaction can only depend on the relative momenta of the incoming and outgoing particles $\mathbf{k}$ and $\mathbf{k}^{\prime}$. The regulator function $\langle\mathbf{u} \mid g\rangle=g(\mathbf{u})=\exp \left(-u^{2} / \Lambda^{2}\right)$ cuts off the contribution of momentum states with $u \gg \Lambda$. The cutoff parameter $\Lambda$ is arbitrary and in the end all observables should be independent of $\Lambda$. The interactions in Eq. (2) are separable and thus, the two-body problem for each partial wave can be solved analytically. The two-body t-matrix can be written as $t_{t, s}(E)=|g\rangle \tau_{t, s}(E)\langle g|$, where $E$ denotes the energy. 
The two-body propagator $\tau_{t, s}(E)$ is given by:

$$
\tau_{t, s}(E)=\left[1 / \lambda_{2}^{t, s}-4 \pi \int \mathrm{d} q q^{2} \frac{g(q)^{2}}{E-q^{2}}\right]^{-1} .
$$

Here and in the following we set $\hbar=m=1$. The coupling constants $\lambda_{2}^{s}$ and $\lambda_{2}^{t}$ can be fixed by demanding that the triplet and singlet scattering length $a_{t}$ and and $a_{s}$ are reproduced correctly by the corresponding t-matrices.

The properties of the triton are determined by the Faddeev equations. To leading order in $\ell /|a|$, all internal orbital angular momenta can be set to zero. Thus, the triton spin $1 / 2$ is built up from the spins of the nucleon only. The triton wave function can be decomposed into Faddeev components. Since we are mainly interested in the binding energies and not in the wave functions, we can eliminate all but one of the components and obtain an equation for remaining component $\psi: \psi=G_{0} t P \psi+G_{0} t G_{0} t_{3}(1+P) \psi$, where $G_{0}$ is the free threeparticle propagator and $P=P_{13} P_{23}+P_{12} P_{23}$ is a permutation operator that generates the omitted Faddeev components; $P_{i j}$ exchanges particles $i$ and $j$. The auxilliary quantity $t_{3}$ is the solution of a Lippmann-Schwinger equation with a $S U(4)$-symmetric three-body contact interaction

$$
V_{3}=\mathcal{P}_{a} \lambda_{3} h\left(u_{1}, u_{2}\right) h\left(u_{1}^{\prime}, u_{2}^{\prime}\right)
$$

only. Here $\mathcal{P}_{a}$ denotes the projector on the total antisymmetric three-body state with total spin $S=1 / 2$ and total isospin $T=1 / 2$ as for example given in [14]. The regulator function $h\left(u_{1}, u_{2}\right)=\exp \left(-\left(u_{1}^{2}+\frac{3}{4} u_{2}^{2}\right) / \Lambda^{2}\right)$ is defined in terms of the familiar Jacobi momenta of the three-body system. The three-body coupling $\lambda_{3}$ must be determined from a three-body observable. This interaction is required in order to renormalize the three-body system and achieve independence of the cutoff parameter $\Lambda$. Its renormalization group behavior is governed by a limit cycle [6, 11]. For large values of $\Lambda$ the running of the coupling constant is described by

$$
\lambda_{3}(\Lambda)=\frac{c}{\Lambda^{4}} \frac{\sin \left(s_{0} \ln \left(\Lambda / L_{3}\right)-\arctan \left(1 / s_{0}\right)\right)}{\sin \left(s_{0} \ln \left(\Lambda / L_{3}\right)+\arctan \left(1 / s_{0}\right)\right)},
$$

where $c \approx 0.016$ is a normalization constant and $s_{0} \approx 1.00624$ is a transcendental number that determines the period of the limit cycle. If the cutoff $\Lambda$ is multiplied by a factor $\exp \left(n \pi / s_{0}\right) \approx(22.7)^{n}$ with $n$ an integer, the three-body coupling $\lambda_{3}$ is unchanged. The three-body parameter $L_{3}$ can be determined directly from observable quantities like the triton binding energy $B_{t}$.

Having expressed all relevant coupling constants in terms of physical observables, we are now ready to compute the binding energies of the four-nucleon system.

The binding energies of a four-body system of nucleons can be determined with the Yakubovsky equations. The four-body wavefunction $\Psi$ is first decomposed into Faddeev components, followed by a second decomposition into so-called Yakubovsky components. In the case of nucleons one ends up with two components $\psi_{A}$ and $\psi_{B}$. In the following, we will consider the $\alpha$-particle with total angular momentum $J=0$ and total isospin $T=0$. The Yakubovsky equations can be written as [15]:

$$
\begin{aligned}
& \psi_{A}=G_{0} t P\left[\left(1-P_{34}\right) \psi_{A}+\psi_{B}\right]+\frac{1}{3}\left(1+G_{0} t_{12}\right) G_{0} V_{3} \Psi \\
& \psi_{B}=G_{0} t \tilde{P}\left[\left(1-P_{34}\right) \psi_{A}+\psi_{B}\right]
\end{aligned}
$$


where $G_{0}$ is the free four-particle propagator and $\Psi$ is the full four-body wave function that can be reconstructed from $\psi_{A}$ and $\psi_{B}$. We solve Eqs. (6) numerically in momentum space. For details of the solution and structure of the above equations, we refer the reader to Refs. [1], 16]. The two-body couplings $\lambda_{2}^{t}$ and $\lambda_{2}^{s}$ are fixed by matching Eq. (3) to reproduce the triplet and singlet scattering lengths $a_{t}$ and $a_{s}$, respectively. Instead of $a_{t}$ one can also use the deuteron binding energy $B_{d}=2.22 \mathrm{MeV}$ together with Eq. (11). The difference between these two methods is higher order in $\ell /|a|$ and thus gives a naive estimate of the error made in the order considered. The three-body parameter $L_{3}$ which is implicit in the three-body interaction $V_{3}$, is fixed from the triton binding energy $B_{t}=8.48 \mathrm{MeV}$. We find $L_{3}=21.2 \mathrm{fm}^{-1}\left(L_{3}=19.8 \mathrm{fm}^{-1}\right)$ if $a_{t}\left(B_{d}\right)$ are used as input.

We now calculate the $\alpha$-particle binding energy $B_{\alpha}$ for different values of the cutoff parameter $\Lambda$. We vary $\Lambda$ in the region where the three-nucleon system has exactly one bound state. For values of $\Lambda$ below $4 \mathrm{fm}^{-1}$ there is a strong cutoff dependence. For values in the range $8 \mathrm{fm}^{-1} \leq \Lambda \leq 10 \mathrm{fm}^{-1}$, however, our results are numerically stable and independent of $\Lambda$ to within $5 \%$. This variation is considerably smaller than the errors from higher orders in $\ell /|a|$ of about $30 \%$. The existence of the plateau for $\Lambda \geq 8 \mathrm{fm}^{-1}$ provides sufficient evidence that the renormalization of the four-nucleon system at leading order in $\ell /|a|$ does not require a four-nucleon force. Renormalization of the the three-nucleon system automatically guarantees renormalization of the four-nucleon system.

For the $\alpha$-particle binding energy, we find $B_{\alpha}=29.5 \mathrm{MeV}$. If the deuteron binding energy $B_{d}$ is used as input instead of the triplet scattering length $a_{t}$, we obtain $B_{\alpha}=26.9 \mathrm{MeV}$. This variation is consistent with the expected $30 \%$ accuracy of a leading order calculation in $\ell /|a|$. Our results agree with the (Coulomb corrected) experimental value $B_{\alpha}^{e x p}=(29.0 \pm 0.1) \mathrm{MeV}$ to within $10 \%$. We conclude that the ground state energy of the $\alpha$-particle can be described by an effective theory with short-range interactions only. Of course, a more refined analysis should include Coulomb corrections. However, the size of these corrections is expected to be smaller than the NLO contribution. Furthermore, corrections due to isospin violation will appear naturally at higher order within the effective theory.

The empirically observed correlation between the binding energies of the triton and the $\alpha$-particle (the Tjon line) follows immediately from the absence of a four-nucleon force at leading order in $\ell /|a|$. The Tjon line is generated by variation of the three-body parameter $L_{3}$, which is different for different $N N$ potentials. In Fig. 11, we show our result for the Tjon line with $a_{s}$ and $B_{d}$ as input (lower line) and $a_{s}$ and $a_{t}$ as two-body input (upper line). Both lines generate a band that gives a naive estimate of higher order corrections in $\ell /|a|$. We also show some calculations using phenomenological potentials [8] and a chiral EFT potential with explicit pions [17, 18]. The Tjon line is a general feature of few-body systems with large scattering length. As a consequence, all calculations with interactions that give a large scattering length should lie close to this line. Different short-distance physics and/or cutoff dependence should only move the results along the Tjon line. This can for example be observed in the NLO results with the chiral potential indicated by the squares in Fig. 1] or in the few-body calculations with the low-momentum $N N$ potential $V_{\text {lowk }}$ carried out in Ref. [19]. The $V_{\text {lowk }}$ potential is obtained from realistic $N N$ interactions by intergrating out high-momentum modes above a cutoff $\Lambda$ but leaving two-body observables (such as the large scattering lengths) unchanged. The results of few-body calculations with $V_{\text {lowk }}$ alone are not independent of $\Lambda$ but lie all close to the Tjon line (cf. Fig. 2 in Ref. [19]). (For a different approach to calculate few-nucleon systems based on a low momentum potential, see Ref. [20] and references therein.) A similar conclusion holds for the study with various 


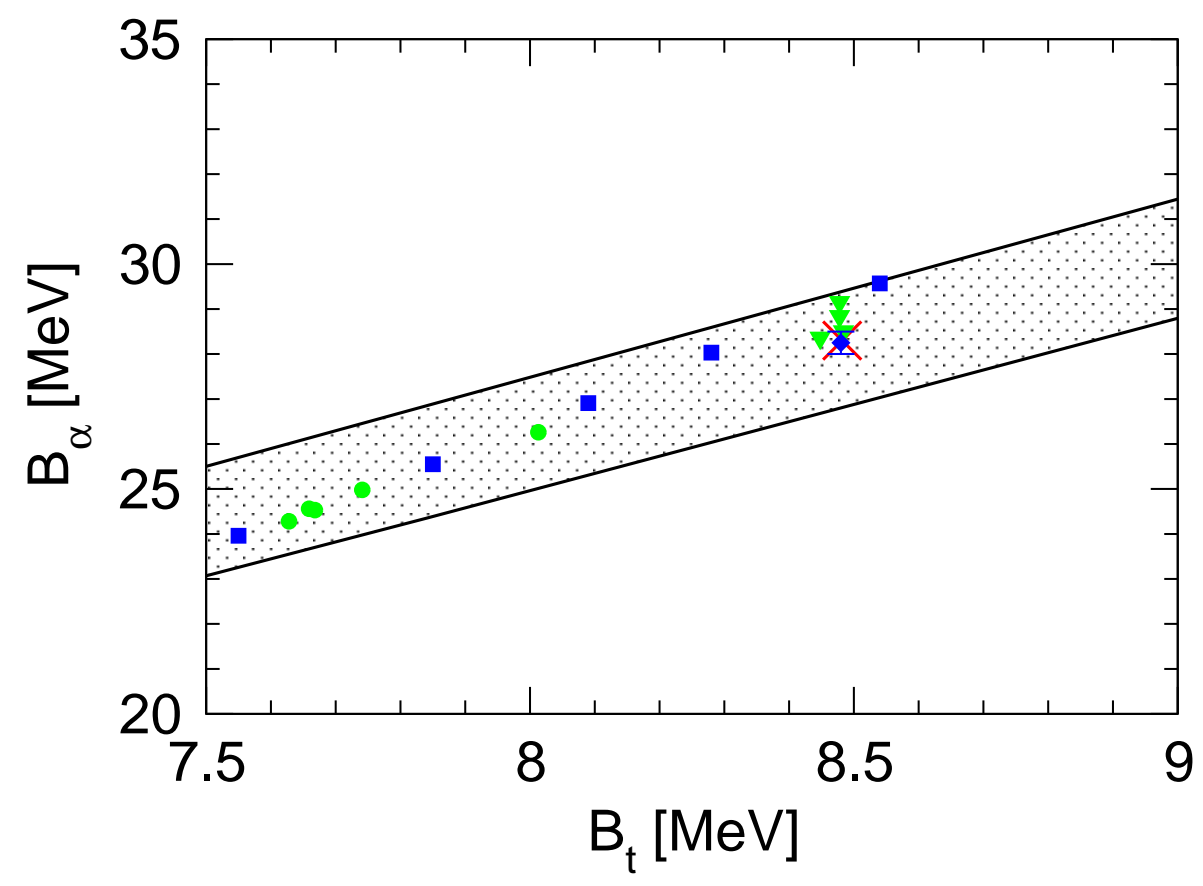

FIG. 1: The correlation between the binding energies of the triton and the $\alpha$-particle (the Tjon line). The lower (upper) line shows our leading order result using $a_{s}$ and $B_{d}\left(a_{s}\right.$ and $a_{t}$ ) as two-body input. The grey dots and triangles show various calculations using phenomenological potentials without or including three-nucleon forces, respectively. The squares show the results of chiral EFT at NLO for different cutoffs while the diamond shows the $\mathrm{N}^{2} \mathrm{LO}$ result. The cross shows the experimental point.

nonlocal potentials in [21]. Our result for the Tjon line to leading order in $\ell /|a|$ is shifted from the empirical values because we neglect finite range effects. Furthermore, the empirical Tjon line becomes a narrow band because of higher order three-nucleon force effects.

In summary, we have computed the $\alpha$-particle binding energy at leading order in the effective theory with contact interactions. Our results are in good agreement with the experimental value and agree within the expected error bounds given by an estimate of higher order contributions. At leading order no four-body force is needed for renormalization and thus, the Tjon line can be understood as a general feature of low-energy systems with large scattering length. As a consequence, all calculations with interactions that give a large scattering length should lie close to this line. Finally, further effort should be devoted to the computation of scattering observables and the inclusion of effective range corrections.

\section{Acknowledgments}

We thank A. Nogga for valuable discussions and comments on the manuscript. L. Platter thanks the INT in Seattle for its hospitality during completion of this work. This research was supported in part by the German Academic Exchange Service (DAAD), the US Department of Energy under grant DE-FG02-00ER41132, and the Deutsche Forschungs- 
gemeinschaft through funds provided to the SFB/TR 16.

[1] V. Efimov, Sov. J. Nucl. Phys. 12, 589 (1971).

[2] E. Braaten and H.-W. Hammer, arXiv:cond-mat/0410417.

[3] P. F. Bedaque and U. van Kolck, Ann. Rev. Nucl. Part. Sci. 52, 339 (2002) arXiv:nucl-th/0203055.

[4] A.C. Phillips, Nucl. Phys. A 107, 209 (1968).

[5] V. Efimov and E.G. Tkachenko, Phys. Lett. 157B, 108 (1985); Sov. J. Nucl. Phys. 47, 17 (1988).

[6] P. F. Bedaque, H.-W. Hammer and U. van Kolck, Nucl. Phys. A 676, 357 (2000) arXiv:nucl-th/9906032.

[7] J.A. Tjon, Phys. Lett. B 56, 217 (1975).

[8] A. Nogga, H. Kamada and W. Glöckle, Phys. Rev. Lett. 85, 944 (2000) arXiv:nucl-th/0004023.

[9] G. P. Lepage, arXiv:nucl-th/9706029.

[10] P. F. Bedaque, H.-W. Hammer and U. van Kolck, Phys. Rev. Lett. 82, 463 (1999) arXiv:nucl-th/9809025; Nucl. Phys. A 646, 444 (1999) arXiv:nucl-th/9811046.

[11] L. Platter, H.-W. Hammer and U.-G. Meißner, Phys. Rev. A 70, 052101 (2004) arXiv:cond-mat/0404313.

[12] U.-G. Meißner, arXiv:nucl-th/0409028.

[13] P. Navratil and E. Caurier, Phys. Rev. C 69, 014311 (2004) arXiv:nucl-th/0311036.

[14] A. Delfino and W. Glöckle, Phys. Rev. C 30, 376 (1984).

[15] W. Glöckle and H. Kamada, Nucl. Phys. A 560, 541 (1993).

[16] L. Platter, H.-W. Hammer and U.-G. Meißner, in preparation.

[17] E. Epelbaum, H. Kamada, A. Nogga, H. Witala, W. Glöckle and U.-G. Meißner, Phys. Rev. Lett. 86, 4787 (2001) arXiv:nucl-th/0007057.

[18] E. Epelbaum, A. Nogga, W. Glöckle, H. Kamada, U.-G. Meißner and H. Witala, Phys. Rev. C 66, 064001 (2002) arXiv:nucl-th/0208023.

[19] A. Nogga, S. K. Bogner and A. Schwenk, arXiv:nucl-th/0405016.

[20] S. Fujii, E. Epelbaum, H. Kamada, R. Okamoto, K. Suzuki and W. Glöckle, Phys. Rev. C 70, 024003 (2004) arXiv:nucl-th/0404049.

[21] R. Lazauskas and J. Carbonell, Phys. Rev. C 70, 044002 (2004) arXiv:nucl-th/0408048. 